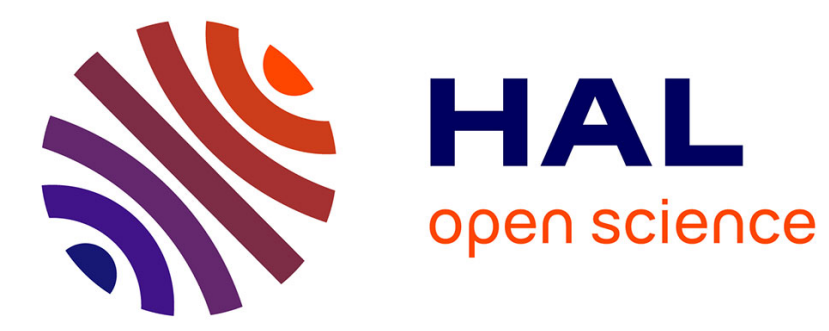

\title{
Magnetic Interactions on Oxide Ferromagnet/Ferromagnetic Intermetallide Interface
}

G. Ovsyannikov, V. Demidov, T. Shaikhulov, A. Klimov, Nicolas Tiercelin, Philippe Pernod, S. Nikitov

\section{- To cite this version:}

G. Ovsyannikov, V. Demidov, T. Shaikhulov, A. Klimov, Nicolas Tiercelin, et al.. Magnetic Interactions on Oxide Ferromagnet/Ferromagnetic Intermetallide Interface. Physics of the Solid State, 2019, 61 (9), pp.1652-1657. 10.1134/S1063783419090191 . hal-02318292

\section{HAL Id: hal-02318292 \\ https://hal.science/hal-02318292}

Submitted on 11 Oct 2020

HAL is a multi-disciplinary open access archive for the deposit and dissemination of scientific research documents, whether they are published or not. The documents may come from teaching and research institutions in France or abroad, or from public or private research centers.
L'archive ouverte pluridisciplinaire HAL, est destinée au dépôt et à la diffusion de documents scientifiques de niveau recherche, publiés ou non, émanant des établissements d'enseignement et de recherche français ou étrangers, des laboratoires publics ou privés. 


\title{
Magnetic Interactions on Oxide Ferromagnet/Ferromagnetic Intermetallide Interface
}

\author{
G. A. Ovsyannikov ${ }^{a, *}$, V. V. Demidov ${ }^{a}$, T. A. Shaikhulov ${ }^{a}$, A. A. Klimov ${ }^{a, b}$, \\ N. Tiercelin ${ }^{c}$, P. Pernod ${ }^{c}$, and S. A. Nikitov ${ }^{a}$ \\ ${ }^{a}$ Kotelnikov Institute of Radio Egineering and Electronics, Russian Academy of Sciences, Moscow, Russia \\ ${ }^{b}$ Russian Technological University MIREA, Moscow, Russia \\ ${ }^{c}$ University of Lille, CNRS, Centrale Lille, ISEN, Univ. Valenciennes, UMR 8520-IEMN, F-59000 Lille, France \\ *e-mail@: gena@hitech.cplire.ru
}

Received April 15, 2019; revised April 22, 2019; accepted April 24, 2019

\begin{abstract}
The magnetic properties of heterostructures consisting of two films are studied. The upper layer involves rare-earth intermetallic nanostructured superlattices consisting of exchange-coupled layers $\left(\mathrm{TbCo}_{2} / \mathrm{FeCo}\right)_{n}$ (TCFC), and the lower layer includes either epitaxial manganite $\mathrm{La}_{0.7} \mathrm{Sr}_{0.3} \mathrm{MnO}_{3}$ (LSMO) with optimum strontium doping or an epitaxial film of an yttrium-iron garnet $\mathrm{Y}_{3} \mathrm{Fe}_{5} \mathrm{O}_{12}$ (YIG) with a Bi additive. TCFC is a ferromagnet having high Curie temperature and provides controllable induced magnetic anisotropy. Experimental studies showed that the interlayer interaction of the TCFC/LSMO heterostructure is antiferromagnetic. There was an increase in the FMR line width in the structures due to the flow of a spin current through the interface between two films. There was electric voltage in the TCFC/YIG heterostructure induced in the TCFC intermetallide film, due to an inverse spin Hall effect under ferromagnetic resonance conditions.
\end{abstract}

Keywords: heterostructure, intermetallic superlattices, manganite, yttrium-iron garnet

DOI: $10.1134 /$ S1063783419090191

\section{INTRODUCTION}

Spintronics is based on phenomena due to the transfer of a spin moment. Spin current can appear via several mechanisms, among which the spin Hall effect [1] and spin pumping during the ferromagnetic resonance are the most effective [2]. A structure, in which spin current is generated, consists of two layers: magnetic and nonmagnetic. The spin current may be detected with an inverse spin Hall effect (ISHE) in a material with strong spin-orbit interaction via converting into conduction current. Not only nonmagnetic metals, however, can be used as ISHE spin current detectors. Some studies showed that magnetic metals, such as $\mathrm{Ni}_{81} \mathrm{Fe}_{19}$ (permalloy), $\mathrm{Fe}, \mathrm{Co}$, and $\mathrm{Ni}$, can be used as spin current detectors [3-5].

$\left(\mathrm{TbCo}_{2} / \mathrm{FeCo}\right)_{n}$ (TCFC) superlattices containing $\mathrm{Tb}$ with a strong spin-orbit interaction (high atomic weight $Z=159)$ possess high Curie temperature $\left(T_{\mathrm{C}}=\right.$ $120^{\circ} \mathrm{C}$ ), giant magnetostriction, controllable magnetic induction anisotropy, and the possibility of inducing spin-orientation transitions by imposing magnetic field or elastic stresses [6, 7]. All these properties of TCFCs suggest that these films may be used for detection of spin current with the inverse spin Hall effect. It is possible that the ferromagnetism in a material with a strong spin-orbit interaction will enhance the magnitude of the current detected.

On the other hand, rare-earth manganite perovskites with a structure $\operatorname{Re}_{1-x} \mathrm{~A}_{x} \mathrm{MnO}_{3}$ (where Re are rare-earth materials, such as $\mathrm{La}$ or $\mathrm{Nd}$, and $\mathrm{A}$ are alkaline earth metals, such as $\mathrm{Sr}, \mathrm{Ca}$, and $\mathrm{Ba}$ ) possess unusual electrical and magnetic properties, including high magnetic polarization up to $100 \%$, great magnetoresistance, etc. $[8,9]$. Manganite films, whose Curie temperature $T_{\mathrm{C}}$ is close to room temperature, is especially attractive for practical purposes. The spin current excitation by ferromagnetic resonance in the LSMO/ $N$ structures ( $\mathrm{LSMO}$ is $\mathrm{La}_{0.7} \mathrm{Sr}_{0.3} \mathrm{MnO}_{3}$ and $\mathrm{N}$ is a normal metal, usually platinum) was studied [10, 11]. These works do not describe the data on temperature dependences of the width of a ferromagnetic resonance (FMR) line during the generation of spin current in ferromagnets and the magnitude of spin conductivity of the LSMO/Pt boundary.

In this work, we study the magnetic properties of rare-earth intermetallic superlattices consisting of exchange-coupled layers $\left(\mathrm{TbCo}_{2} / \mathrm{FeCo}\right)_{n}$ and either LSMO manganite epitaxially grown or epitaxial $\mathrm{Y}_{3} \mathrm{Fe}_{5} \mathrm{O}_{12}$ (YIG) yttrium-iron garnet film with a $\mathrm{Bi}$ additive. We especially focus on the effects that 


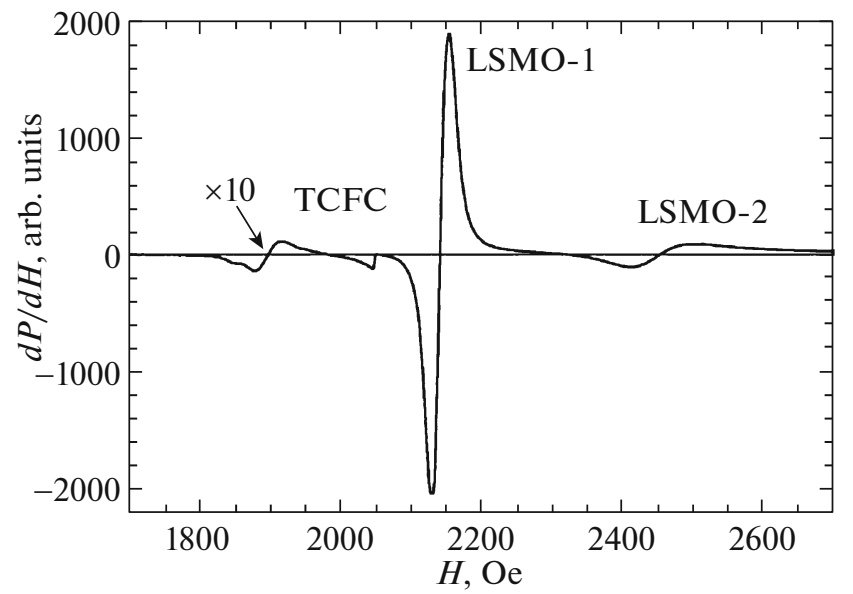

Fig. 1. Ferromagnetic resonance spectrum for TCFC LSMO heterostructure acquired at room temperature and with the direction of external magnetic field along the axis of easy magnetization. The amplitude of low-field line (FMR of TCFC) increased tenfold compared to the other two lines.

appeared due to excitation and generation of spin current in the structures.

\section{FERROMAGNETIC RESONANCE IN TCFC/LSMO HETEROSTRUCTURE}

FMR spectrum of the TCFC/LSMO heterostructure (Fig. 1) was acquired at the frequency $\omega / 2 \pi=$ $9.74 \mathrm{GHz}$ and $T=300 \mathrm{~K}$ with a constant magnetic field lying in the plane of the substrate and directed along the axis of easy magnetization of TCFC. There are three FMR lines attributable to the three ferromagnetically ordered spin systems. At the same time, LSMO films were epitaxially grown via magnetron sputtering at high temperature on a $\mathrm{NdGaO}_{3}$ substrate of $5 \times 5 \mathrm{~mm}^{2}$ in size, whereas TCFC superlattices were obtained via layer-by-layer magnetron sputtering of $\mathrm{TbCo}_{2}$ and FeCo layers in a magnetic field setting anisotropy. The TCFC superlattice occupied only part of the substrate.

The temperature dependences of resonant fields of these three lines (Fig. 2) showed that the line at 1900 Oe (Fig. 1) is due to TCFC superlattice, which has a higher Curie temperature compared to that of LSMO. Two other lines are assigned to LSMO film: LSMO-2 corresponds to the part of LSMO film lying under the TCFC superstructure, whereas LSMO-1 is assigned to that not covered with TCFC film. The number of electron spins for the LSMO-1 and LSMO2 peaks is determined by the topology of a sample (the area covered by TCFC and not covered by the part of the substrate). The number of spins was assessed via calculation of the area of absorption line in FMR spectrum.

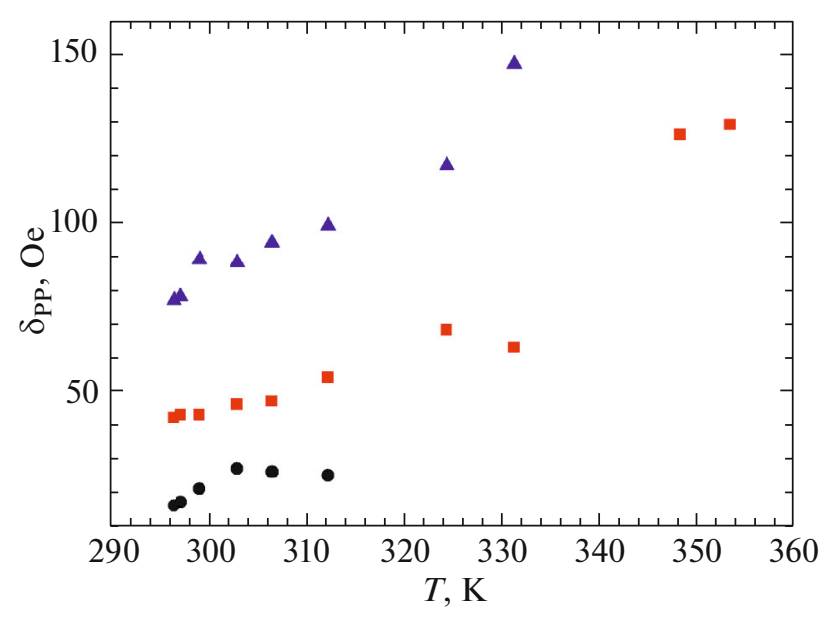

Fig. 2. Temperature dependences of the widths of FMR lines $\left(\delta_{\mathrm{PP}}\right)$ with the direction of external magnetic field along the axis of difficult magnetization for different parts of heterostructure. Triangles and rectangles designate LSMO film, whereas circles designate TCFC.

The spin precession decay $\alpha_{0}=\delta_{\mathrm{PP}} \gamma / \omega=12 \times 10^{-3}$ was found at room temperature for the LSMO films from the FMR line width $\delta_{\mathrm{PP}}=40 \mathrm{Oe} . \delta_{\mathrm{PP}}$ of the two lines of LSMO-1 and LSMO-2 films differ by 4050 Oe. Considering that both parts of LSMO-1 and LSMO-2 are located on the same substrate and have the same crystal structure, this difference in the widths of FMR lines is probably due to interaction between the TCFC superlattice and LSMO film. An Increase in attenuation $\alpha$ during TCFC film spraying on LSMO $\alpha=\alpha_{0}+\alpha$ may arise due to the spin current through the TCFC/LSMO interface [12]. If the thickness of TCFC film deposited on LSMO is $10 \mathrm{~nm}$, the parameter $\alpha$ increases by two times at room temperature (Fig. 2). The spin conductivity in the TCFC/LSMO heterostructure may be calculated as follows [13, 14]:

$$
g_{\text {eff }}^{\uparrow \downarrow}=\frac{4 \pi M_{s} t_{\text {LSMO }}}{g \mu_{\mathrm{B}}} \alpha^{\prime},
$$

where $\gamma=17.605 \times 10^{6}$ is the gyromagnetic ratio for an electron, $\omega=2 \pi \times 9.51 \times 10^{9} \mathrm{~s}^{-1}$ the angular frequency, $M_{s}=300$ Oe the magnetization of LSMO film, $t_{\mathrm{LSMO}}=40 \mathrm{~nm}$ the thickness of LSMO film, $\mu_{\mathrm{B}}=$ $9.274 \times 10^{-21} \mathrm{erg} / \mathrm{G}$ the Bohr magneton, and $g=2$ the Lande factor. The width of FMR line increased at room temperature after the TCFC film was deposited: $\delta_{\mathrm{TCFC} / \mathrm{LSMO}}-\delta_{\mathrm{LSMO}}=40 \mathrm{Oe}$, so that $g_{\mathrm{eff}}=9.7 \times$ $10^{19} \mathrm{~m}^{-2}$. This spin conductivity value of the boundary exceeds that of $g_{\text {eff }}=5 \times 10^{18} \mathrm{~m}^{-2}$ obtained from measurements of spin current on the Pt/LSMO structures using the inverse spin Hall effect [15]. For comparison, $g_{\text {eff }}$ for the Py/Pt and YIG/Pt boundaries were $2.1 \times 10^{19}[16]$ and $4.8 \times 10^{20} \mathrm{~m}^{-2}$ [17], respectively. 


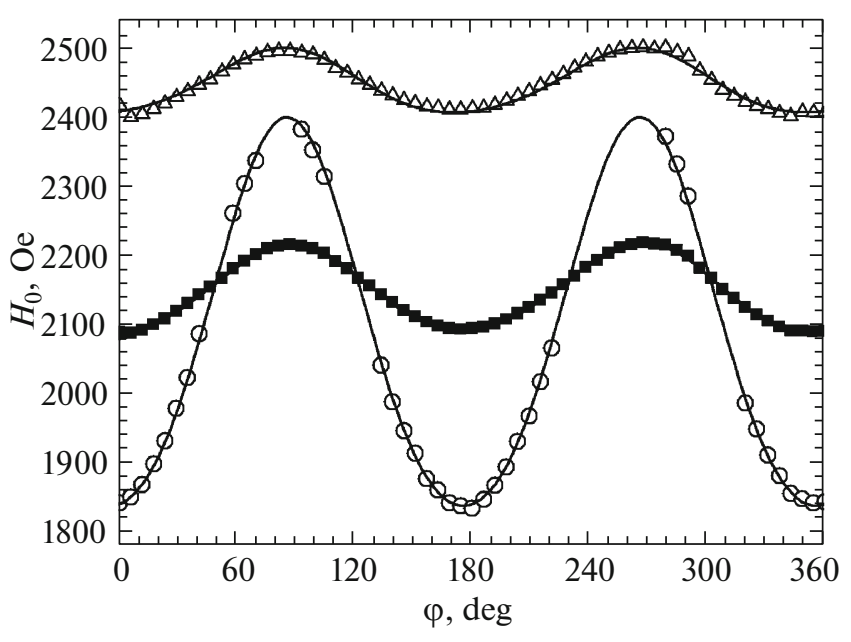

Fig. 3. Angular dependence of resonant fields of three FMR lines for TCFC/LSMO heterostructure. Solid lines show the calculated dependencies. Triangles and rectangles designate LSMO film, whereas circles show TCFC.

Figure 2 shows the temperature dependences of FMR line widths, when the external magnetic field is directed along the axis of difficult magnetization for different parts of the heterostructure. The triangles and rectangles belong to LSMO-1 and LSMO-2 films, respectively, whereas circles are attributable to TCFC superlattice. It is clear that the widths of FMR lines for the LSMO-1 and LSMO-2 films increase with an increase in temperature, and the difference $\delta_{\mathrm{TCFC} / \mathrm{LSMO}}-\delta_{\mathrm{LSMO}}$ is slightly changed within the temperature range of 300-320 K. It should be noted that there is a uniaxial magnetic anisotropy in LSMO films, which also leads to decay anisotropy. Considering that magnetic anisotropies of the LSMO-1 and LSMO-2 films have the same angular dependence (Fig. 3), the same direction of the external magnetic field should be used in assessment of spin conductivity $g_{\text {eff }}$.

The solution of Landau-Lifshitz-Hilbert equation gives two resonance relationships $\omega\left(H_{0}\right)$, which describe the FMR in TCFC and LSMO-2 films, taking into account the uniaxial and biaxial anisotropies. These relationships are similar to those obtained in [18] for an autonomous LSMO film deposited on a (110) $\mathrm{NdGaO}_{3}$ orthorhombic substrate, which causes the uniaxial magnetic anisotropy [19]. The $H_{0}$ value should be replaced by the sum of the two terms $H_{01}+$ $H_{J 1}$ and $H_{02}+H_{J 2}$ for the LSMO-2 film and TCFC superlattice, respectively, to take into account the magnetic interaction in the equations for resonant frequency $\omega\left(H_{0}\right)$ [19]. In this case, $H_{01}$ and $H_{02}$ are resonant fields values for LSMO-2 and TCFC, respectively. $H_{J 1}=J /\left(M_{1} d_{1}\right)$ and $H_{J 2}=J /\left(M_{2} d_{2}\right)\left(d_{1}\right.$ and $d_{2}$ are the thicknesses of LSMO and TCFC layers, respectively) give an effective interlayer exchange

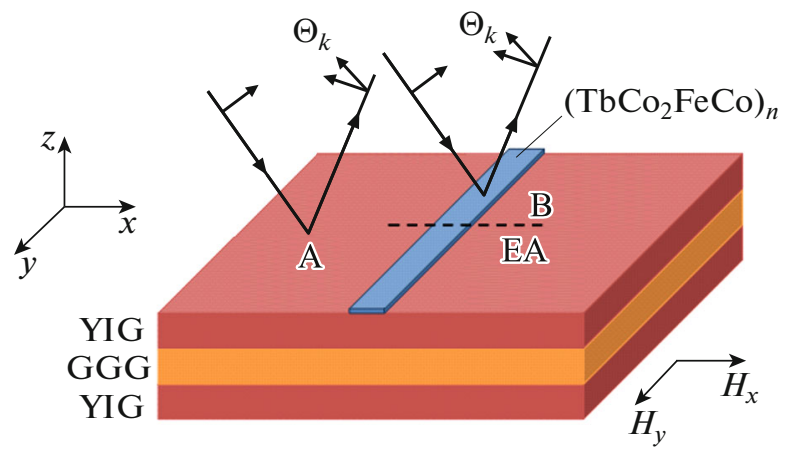

Fig. 4. Schematic representation of heterostructure in the Kerr effect geometry. " $\mathrm{A}$ " and "B" indicate the position of a laser beam for TCFC/YIG heterostructure and YIG film.

interaction for the LSMO-2 and TCFC films with an interaction constant $J$.

The angular dependence of FMR response of an autonomous LSMO film (LSMO-1) (Fig. 3) is firstly calculated to find the structure parameters. Then the angular dependence of $H_{0}$ is calculated for the LSMO2 film from the magnetization magnitude obtained by fitting the relationship between resonant field and angle. Then the exchange interaction constant $J$ is found. Finally, the $J$ value obtained is used to calculate the angular dependence of $H_{0}$ for the TCFC film and to calculate $M_{2}$ value. As a result, the data obtained allow us to conclude that the TCFC/LSMO interface possesses the antiferromagnetic interlayer interaction with a constant $J=-0.24 \mathrm{erg} / \mathrm{cm}$.

\section{SPIN CURRENT IN TCFC/ $\mathrm{Y}_{3} \mathrm{Fe}_{5} \mathrm{O}_{12}$ HETEROSTRUCTURE}

Yttrium-iron garnet (YIG) films are very attractive for spintronic structures due to their low magnetic attenuation and low conductivity. The spin current can be excited in a YIG film and detected with a permalloy film [3-5].

An epitaxial film of an $\mathrm{Y}_{3} \mathrm{Fe}_{5} \mathrm{O}_{12}$ (YIG) garnet doped with $\mathrm{Bi}$ and with $4 \mu \mathrm{m}$ thickness was grown on a (111) $\mathrm{Gd}_{3} \mathrm{Ga}_{5} \mathrm{O}_{12}(\mathrm{GGG})$ substrate. A superlattice of TCFC intermetallic compounds with a thickness of $30 \mathrm{~nm}$, which covered only part of the substrate (Fig. 4), was deposited on it. The magnetic parameters of a heterostructure-the coercive force $\left(H_{\mathrm{C}}\right)$ and the saturation field $\left(H_{\mathrm{S}}\right)$-were studied with a meridional Kerr effect [19]. The sample was placed in the electromagnet gap in the magnetization directions along and across the axes of easy (ea) and hard (ha) magnetic axes of the TCFC layer. The measurements were performed by focusing the laser beam either at the point "A" or "B" (Fig. 4), which corresponds to measurements of either YIG film or the TCFC/YIG hetetrostructure. 


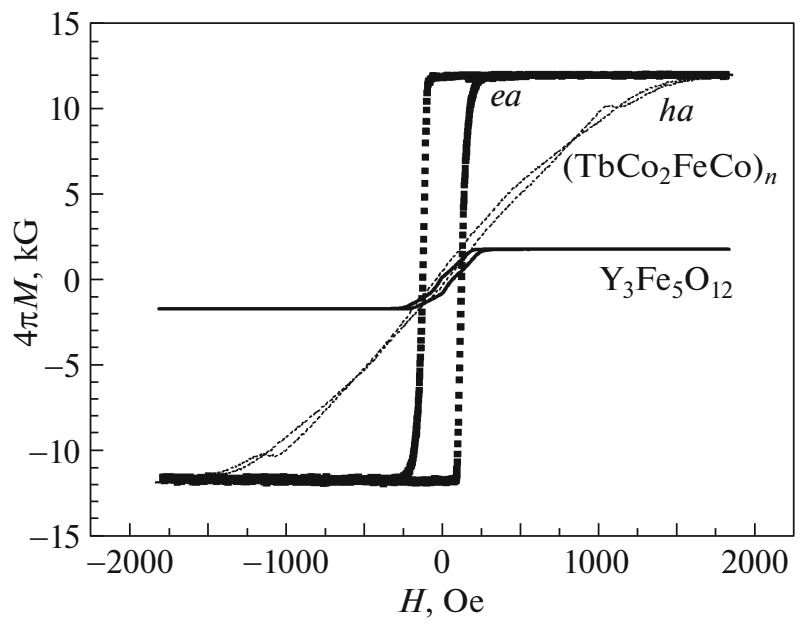

Fig. 5. Magnetization curves of TCFC/YIG heterostructure and YIG films acquired upon the direction of external magnetic field along easy and hard magnetic magnetization axes.

Figure 5 shows the dependencies of magnetic induction of the YIG film and the TCFC/YIG heterostructure on the magnetic field obtained with Kerr effect for two directions: along the hard and easy magnetization axes. The measurements for the TCFC/YIG heterostructure were carried out with a test laser beam in the B position (Fig. 4). In this case, there is no influence of the lower YIG film on the magnetization of the heterostructure, because the laser beam is shielded by conduction currents flowing in the TCFC film. The induction of saturations of the YIG and TCFC films were taken from literature data $[20,21]$. The magnetooptical response from the lower YIG film was measured at the position of the beam at the A point (Fig. 4). It is clear that the saturation field of the heterostructure (actually, a TCFC film) $H_{\mathrm{S}} \approx$ 1500 Oe significantly exceeds that of the YIG film $\left(H_{\mathrm{S}} \approx 200 \mathrm{Oe}\right)$. When the direction of the external magnetic field changes, there is a clear magnetization anisotropy for the TCFC film. At the same time, there was no magnetization anisotropy for the YFO film.

The ferromagnetic resonance of a structure was measured with a strip line in two modes: by registering an external signal via modulation in a magnetic field and modulation by an external microwave signal together with the transmission and reflection modes of the microwave signal. The substrate with a film was pressed against a strip line. Figure 6 shows the dependence of a $S_{12}$ transmission coefficient of a strip line with a YIG film at six external exposure frequencies on magnetic field. It is clear that when the frequency changes, the line shape remains unchanged. There was no a sharp change in line width with a frequency in the range measured. There was no absorption line at frequencies below $1 \mathrm{GHz}$, probably because of ferromagnetic domains in the YIG film.

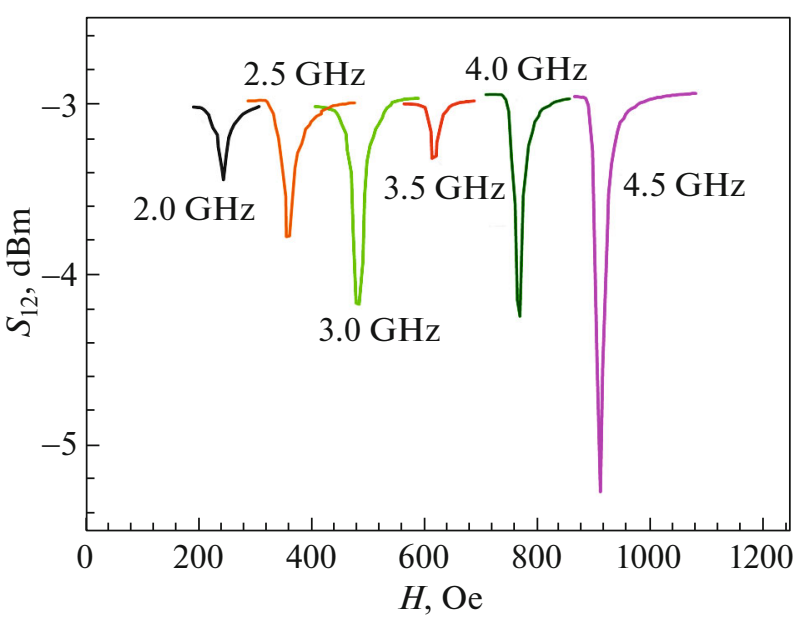

Fig. 6. Ferromagnetic resonance spectra (transmission coefficient $S_{12}$ ) of YIG film under the action of microwave radiation at six frequencies of external influence.

The derivative of a reflection coefficient $d P / d H$ is measured in the modulation mode of magnetic field. Figure 7 shows the $d P / d H(H)$ dependences separately for the YIG film and TCFC/YIG heterostructure. The FMR spectrum of a YIG film consists of two lines obtained by fitting the spectrum with two Lorentz lines. Two lines in the FMR spectrum are probably due to preparation of a YIG film. The crystallographic difference has stronger effect near the substrate, and the quality of the film is worse (worse inhomogeneity, lower magnetization, and greater FMR width). Then the layers become more uniform, and the outer part of the film, with which the TCFC contacts, has the best uniformity. As a result, an FMR line with a smaller

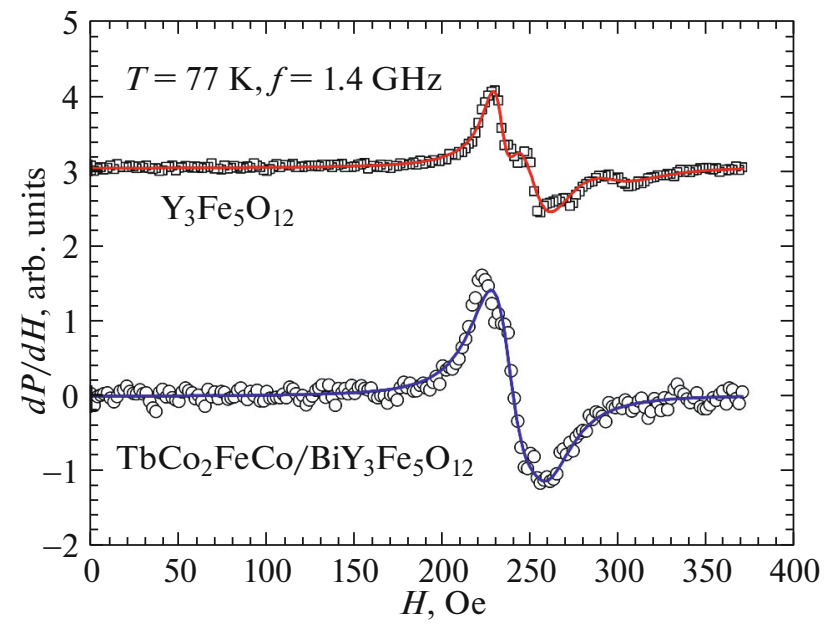

Fig. 7. FMR spectra $(d P / d H)$ of: (1) YIG film and (2) TCFC/YIG heterostructures obtained for reflection in the modulation mode of external magnetic field. $f=$ $1.4 \mathrm{GHz}$ and $T=77 \mathrm{~K}$. 


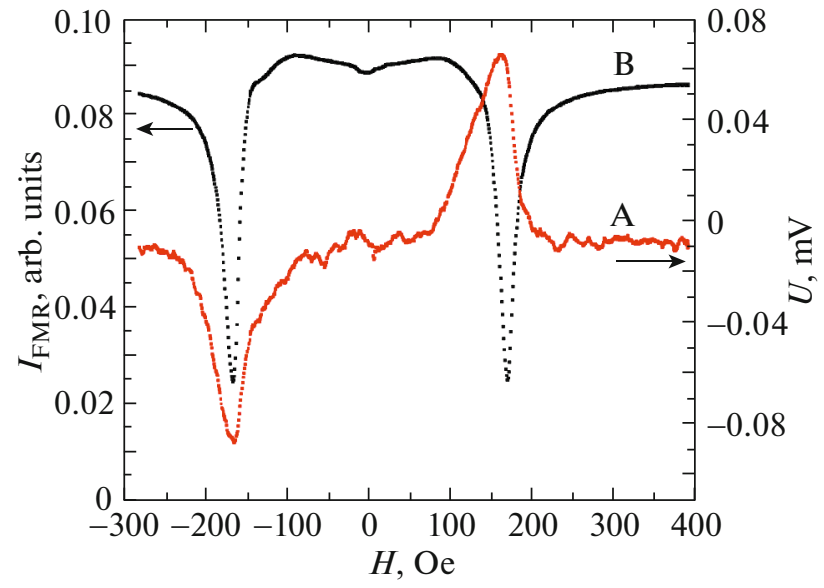

Fig. 8. The signal of inverse spin Hall effect arising on TCFC/YIG heterostructure in the modulation mode of amplitude of external microwave field.

resonant field (greater magnetization) was chosen to calculate the parameters of spin current. The width of FMR line of TCFC film was chosen to assess the spin conductivity of TCFC/YIG boundary, because the YIG layer adjacent to the TCFC participates in the formation of spin current. There was a broad FMR line due to TCFC film over the YIG one. The spin precession decay $\alpha_{0}$ at the nitrogen temperature may be found for YIG films from the line width having the minimum value $\delta_{\mathrm{PP}}=9 \mathrm{Oe}$, which corresponds to $\alpha_{0}=\Delta \delta_{\mathrm{PP}} \gamma / \omega=18 \times 10^{-3}$. The spin conductivity of the boundary in TCFC/YIG heterostructure may be calculated according to Eq. (1) with $\omega=2 \pi \times 1.4 \times 10^{9} \mathrm{~s}^{-1}$, $M_{s}=138$ Oe (magnetization for LSMO film), and $t_{\mathrm{YIG}}=4 \mu \mathrm{m}$ (the thickness of YIG film) [13, 14]. An increase in FMR line width was obtained at nitrogen temperature after deposition of TCFC by $10 \mathrm{Oe}$ and, therefore, $g_{\text {eff }}=7.5 \times 10^{21} \mathrm{~m}^{-2}$. This spin conductivity value of the boundary is significantly higher than $g_{\text {eff }}=$ $9.7 \times 10^{19} \mathrm{~m}^{-2}$ obtained from measurements of an FMR line width of TCFC/LSMO heterostructures (Section 2). Higher spin conductivity value is probably due to a large thickness of the YIG film $t_{\mathrm{YIG}}=4 \mu \mathrm{m}$ used in the calculation according to Eq. (1). Only the upper part of the YIG film probably participates in the generation of spin current.

Figure 8 shows the experimental results of inverse spin Hall effect ISHE in the TCFC/YIG heterostructure. The contact areas were formed on the surface of the heterostructure to measure the potential difference caused by ISHE effect. The spin current $\left(J_{S}\right)$ may be detected from inverse spin Hall effect in a material with strong spin-orbit interaction via transformation into conduction current $J_{c}=\theta_{\mathrm{SH}}(\hbar / 2 e)\left(J_{S} \sigma\right)$, where $\theta_{\mathrm{SH}}$ is the angle of spin Hall effect and $\sigma$ the polarization of carriers. When the external magnetic field was changed, the potential difference interfaces and the intensity of an FMR signal were recorded. Figure 8 shows the FMR spectrum of the YIG film acquired in a microstrip configuration at a frequency of $1615 \mathrm{MHz}, T=300 \mathrm{~K}$, a generator power of $2 \mathrm{~mW}$, and in magnetic field in the substrate plane directed along the axis of the difficult magnetization of TCFC material. When $H$ was $169 \pm 0.2$ Oe, there was a peak due to FMR in the YIG film. There is a slight asymmetry of the peak position relative to a change in polarity of the magnetic field. The half width of the peak is 24 Oe. There is no the second peak caused by TCFC ferromagnetic layer due to its small thickness. Figure 8 also shows the field dependence of voltage due to inverse spin Hall effect measured on the TCFC film. There is a strong asymmetry of ISHE peak of $\sim 7 \mathrm{Oe}$. The width of ISHE peak is $60 \mathrm{Oe}$. When the direction of magnetization field was changed, there was a change in sign of ISHE voltage, whose value was $80 \mu \mathrm{V}$.

\section{CONCLUSIONS}

Experimental studies of magnetic properties of a heterostructure consisting of manganite LSMO grown epitaxially and coated with a rare-earth intermetallic TCFC superlattice showed that the magnetic interaction in the heterostructure is antiferromagnetic. The energy of exchange interaction of the layers in the heterostructure was found. There was an increase in line width of ferromagnetic resonance due to spin current flowing through the TCFC/LSMO boundary. The spin conductivity of boundaries was assessed from the measurements of line width of ferromagnetic resonances in the TCFC/LSMO and TCFC/YIG heterostructures. An electrical voltage was experimentally observed due to inverse spin Hall effect in a TCFC film under ferromagnetic resonance conditions in YIG.

\section{ACKNOWLEDGMENTS}

The authors are grateful to K.I. Constantinian, A.M. Petrzhik, V.L. Preobrazhenskii, and A.V. Shadrin for their useful discussion of the results and assistance in this study.

\section{FUNDING}

This work was performed within the framework of the state task and it was partially supported by the Russian Foundation for Basic Research (projects 18-57-16001, 1907-00143, and 17-02-00145), and the LEMAC-LICS International Laboratory.

\section{CONFLICT OF INTEREST}

There are no conflicts of interest to declare. 


\section{REFERENCES}

1. M. I. Dyakonov and V. I. Perel, Phys. Lett. A 35, 459 (1971).

2. E. Saitoh, M. Ueda, H. Miyajima, and G. Tatara, Appl. Phys. Lett. 88, 182509 (2006).

3. B. F. Miao, S. Y. Huang, D. Qu, and C. L. Chien, Phys. Rev. Lett. 111, 066602 (2013).

4. P. Hyde, Bai Lihui, D. M. J. Kumar, B. W. Southern, C.-M. Hu, S. Y. Huang, B. F. Miao, and C. L. Chien, Phys. Rev. B 89, 180404(R) (2014).

5. F. Yang and P. C. Hammel, J. Phys. D 51, 253001 (2018).

6. Y. Gall, J. Ben, F. Socha, N. Tiercelin, V. Preobrazhensky, and P. Pernod, J. Appl. Phys. 87, 5783 (2000).

7. E. Quandt, A. Ludwig, D. G. Lord, and C. A. Faunce, J. Appl. Phys. 83, 7267 (1998).

8. A. M. Haghiri-Cosnet, and J. P. Renard, J. Phys. D 36, R127 (2003).

9. N. G. Bebenin, R. I. Zainullina, and V. V. Ustinov, Phys. Usp. 61, 719 (2018).

10. G. Y. Luo, C. R. Chang, and J. G. Lin, J. Appl. Phys. 115, 17C508 (2014).

11. V. A. Atsarkin, B. V. Sorokin, I. V. Borisenko, V. V. Demidov, and G. A. Ovsyannikov, J. Appl. Phys. D 49, 125003 (2016).

12. Ya. Tserkovnyak, A. Brataas, and G. E. W. Bauer, Phys. Rev. Lett. 88, 117601 (2002).
13. G. Y. Luo, M. Belmeguenai, Y. Roussigne, C. R. Chang, J. G. Lin, and S. M. Cherif, AIP Adv. 5, 097148 (2015).

14. J.-C. Rojas-Sanchez, N. Reyren, P. Laczkowski, W. Savero, J.-P. Attane, C. Deranlot, M. Jamet, J.-M. George, L. Vila, and H. Jaffrès, Phys. Rev. Lett. 112, 106602 (2014).

15. V. A. Atsarkin, I. V. Borisenko, V. V. Demidov, and T. A. Shaikhulov, J. Phys. D 51, 245002 (2018).

16. O. Mosendz, V. Vlaminck, J. E. Pearson, F. Y. Fradin, G. E. W. Bauer, S. D. Bader, and A. Hoffmann, Phys. Rev. B 82, 214403 (2010).

17. T. M. Rezende, R. L. Rodriguez-Suarez, M. M. Soares, L. H. Vilela-Le, D. L. Dominguez, and A. Azeved, Appl. Phys. Lett. 102, 012402 (2013).

18. V. V. Demidov, G. A. Ovsyannikov, A. M. Petrzhik, I. V. Borisenko, A. V. Shadrin, and R. Gunnarsson, J. Appl. Phys. 113, 163909 (2013).

19. G. A. Ovsyannikov, A. M. Petrzhik, I. V. Borisenko, A. A. Klimov, Yu. A. Ignatov, V. V. Demidov, and S. A. Nikitov, J. Exp. Theor. Phys. 108, 48 (2009).

20. V. F. Shkar', E. I. Nikolaev, V. N. Sayapin, A. I. Linnik, V. P. Denisenkov, A. M. Grishin, and S. I. Khartsev, Phys. Solid State 47, 1107 (2005).

21. A. S. Grishin, G. A. Ovsyannikov, A. Klimov, V. V. Demidov, K. Y. Constantinian, I. V. Borisenko, V. L. Preobrazhensky, N. Tiercelin, and P. Pernod, J. Electron. Mater. 47, 1595 (2018).

Translated by A. Tulyabaev 